\title{
Enantioselective synthesis of the side-chain acid of homoharringtonine and harringtonine from the same $\gamma$-butyrolactone intermediate
}

\author{
Moran Sun, Yinchao Li, Xiufang Shi, Changling Niu, and Hua Yang* \\ School of Pharmaceutical Science, Zhengzhou University, Zhengzhou, 450001, China \\ E-mail:yanghua@zzu.edu.cn
}

DOI: http://dx.doi.org/10.3998/ark.5550190.p009.388

\begin{abstract}
A versatile intermediate $\gamma$-butyrolactone 7 was prepared from L-alanine with $96 \%$ ee value, which could be used as a common intermediate for the preparation of the side-chain acid of homoharringtonine and harringtonine in $26.6 \%$ and $27 \%$ overall yield respectively. The key steps involved [2, 3]-Meisenheimer rearrangement, allylic bromination, and Barbier reaction. Some interesting experimental phenomena and effective solutions to these issues are reported herein.
\end{abstract}

Keywords: homoharringtonine, harringtonine, [2,3]-Meisenheimer rearrangement, allylic bromination, $\gamma$-butyrolactone

\section{Introduction}

Homoharringtonine (HHT) and its homologue harringtonine (HT) have potent antileukemic activities. In particular, HHT has reached phase III clinical trials in the United States against chronic myelogenous leukemia, while in China, it is used as a front-line therapy for acute myeloid leukemia and shows activity against the chloroquinine-resistant Plasmodium falciparum malaria parasite in vitro. ${ }^{1}$ Because the inactive cephalotaxine (Figure 1) can compose as much as ca. $50 \%$ of total alkaloid extracts, the most practical path for obtaining large quantities of HHT and HT at the present time is through semi-synthesis by coupling the carboxyl group of the respective ester side chain to the C-3 hydroxyl group of cephalotaxine. Several groups have reported enantioselective synthesis of the side-chains of HHT and HT. ${ }^{1-8}$ We have also developed an efficient strategy to construct chiral tertiary alcohol via [2,3]-Meisenheimer rearrangement. Through this strategy, the $(\boldsymbol{R})$-20, side-chain acid of homoharringtonine, and $(\boldsymbol{R})$ 30, side-chain acid of harringtonine, were enantioselectively prepared from $L$-aspartic acid and $L$-threonine respectively. ${ }^{9}$ Obviously, the linear and parallel synthesis of $(\boldsymbol{R})-\mathbf{2 0}$ and $(\boldsymbol{R})-\mathbf{3 0}$ require two different $\alpha$-amino acids as starting materials and two sets of different reagents 
(Figure 1). Considering the economic cost and manpower cost in future laboratory preparation and possible industrial-scale production, we explore a divergent synthesis for $(\boldsymbol{R})-\mathbf{2 0}$ and $(\boldsymbol{R})-\mathbf{3 0}$ from the same intermediate $\gamma$-butyrolactone 7 .
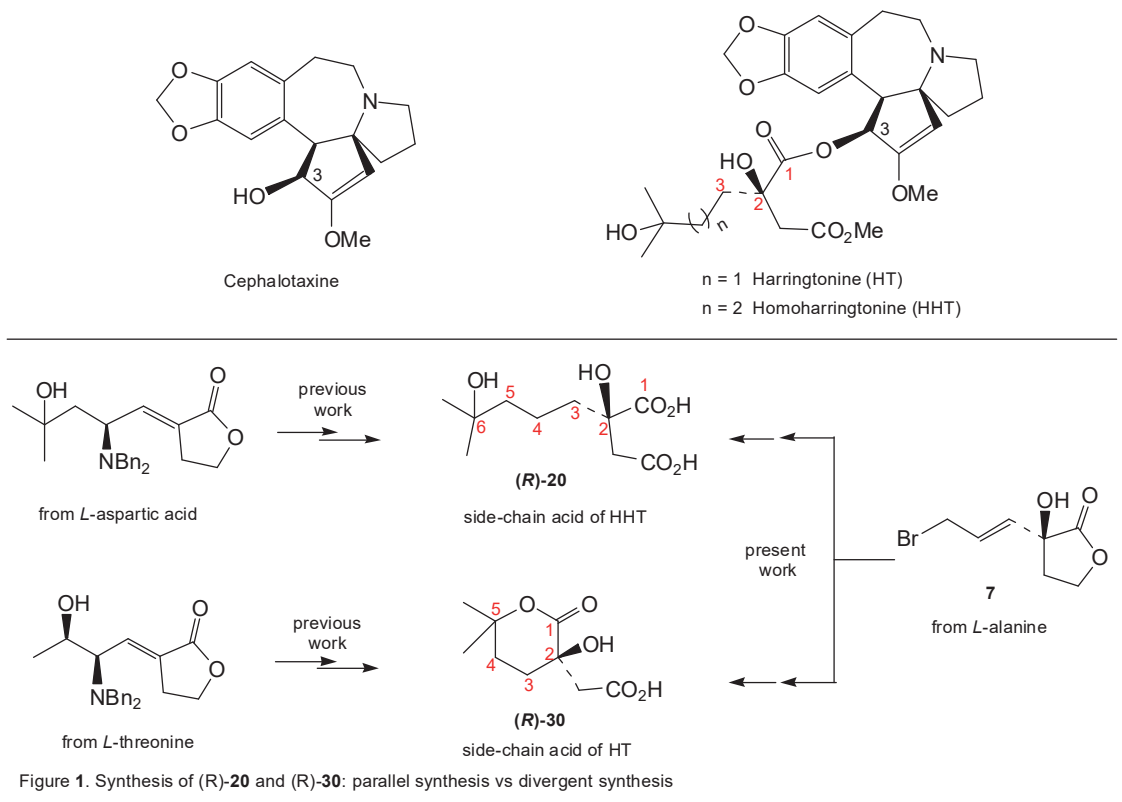

\section{Results and Discussion}

The first step towards key intermediate 7 (Scheme 1) commenced with the reduction of $N$-Boc- $L$ alanine methyl ester 1, which was derived from $L$-alanine. ${ }^{10} \mathrm{~N}$-Boc- $L$-ala-OH 2 was delivered under $\mathrm{NaBH}_{4}-\mathrm{CaCl}_{2}$-THF-EtOH condition ${ }^{11}$ in excellent yield and optical purity $\left(96 \%,[\alpha]^{20}{ }_{\mathrm{D}}-\right.$ 11.4 (c $\left.0.99, \mathrm{CHCl}_{3}\right) ;$ lit. $\left.^{12}[\alpha]^{20} \mathrm{D}-11.6\left(\mathrm{c} 0.6, \mathrm{CHCl}_{3}\right)\right)$. 2 were originally subjected to Swern condition ${ }^{13}$ and $N$-Boc-alaninal 3 was readily obtained as white solid. For the obtained aldehyde 3 an $[\alpha]^{20}{ }_{\mathrm{D}}=-4.3(\mathrm{c} 0.8, \mathrm{MeOH})$ was measured, which is lower than that record of literature $[\alpha]^{20} \mathrm{D}=35.2(\mathrm{c} 1, \mathrm{MeOH}){ }^{14}$. This result revealed that serious racemization had occurred during Swern oxidation or subsequent flash column chromatography. To avoid the undesired decomposition and racemization of aldehyde $\mathbf{3}$, the obtained crude product were used immediately to couple with 1-butyrolactonylidene triphenylphosphorane in dry dichloromethane, delivering (E)-olefin 4 as the only isolated product (95\% yield for the two-step sequence). Unexpectedly, $(E)$-olefin 4 had only 34\% ee. After several trials, we observed that careful manipulation under rigorous conditions had a crucial influence on the ee value, which fluctuate between $30 \%$ and $80 \% .{ }^{15}$ Furthermore, it was verified by us that the corresponding aldehyde of Boc- $L$-Leu-OH and Boc- $L-V a l-O H$ don't racemize under the same Swern conditions. Accordingly, we have to sough to Parikh-Doering oxidization, which was reported to oxidize $\mathrm{N}$ Boc- $L$-ala-OH 2 to 3 with no racemization ${ }^{16}$. Eventually, aldehyde 3 was acquired with $99 \%$ ee 
and applied into Wittig reaction without purification by column chromatography. It is worth of note that $(E)$-olefin 4 was obtained as white solid under Parikh-Doering conditions while under Swern conditions as colorless liquid.

Removal of the Boc protecting group followed by doubly benzylated ${ }^{9}$ in $\mathrm{CH}_{3} \mathrm{CN}$ afforded $(E)$-olefin $\mathbf{5}^{17}\left(99 \%\right.$ ee) in $85 \%$ yield over two steps. Treatment of $\mathbf{5}$ with $\mathrm{m}$-CPBA at $5{ }^{\circ} \mathrm{C}$ immediately provide unstable $[2,3]$-Meisenheimer rearrangement product 5a. ${ }^{9}$ Cleavage of $\mathrm{N}-\mathrm{O}$ bond appear to be a challenge due to the instability of 5a. Common used methods such as $\mathrm{Al}$ $(\mathrm{Hg}),{ }^{18} \mathrm{Na}$ (liq. $\left.\mathrm{NH}_{3}\right),{ }^{19} \mathrm{CuSO}_{4}{ }^{20}, \mathrm{TMSI}^{21}$ and $\mathrm{Zn}(\mathrm{HOAc})^{22}$ proved fruitlessness at room temperature, and $\mathrm{Zn}$ (aq. $\mathrm{HCl}-\mathrm{MeOH})^{23}$ provided 3\%-5\% racemized 6. The troublesome racemization was finally circumvented by addition of $\mathrm{HOAc}$ to $\mathrm{Zn}$ (aq. $\mathrm{HCl}-\mathrm{MeOH}$ ) reduction system, which reduced the acidity of reductive system and so the risk of racemization was depressed. 6 could be obtained in $86 \%$ yield with $96.3 \%$ ee and only about $1.5 \%$ level of racemization occurred.

During Wohl-Ziegler bromination ${ }^{24}$ of compound 6, routine monitoring technique did not work because that 6 and 7 had identical $R_{\mathrm{f}}$ values, and moreover, product 7 was visualized under $254 \mathrm{~nm}$ violet lamp while $\mathbf{6}$ were not. One simple technique has been developed to monitor such reactions. While the reaction proceed, just take a small quantity of solution, concentrate under reduced pressure, add $\mathrm{CH}_{3} \mathrm{CN}(0.5 \mathrm{~mL})$ and aqueous $\mathrm{AgNO}_{3}(10 \%)$ sucessively, shake violently for a few minutes, then monitor the produced solution by TLC. Since bromide 7 can be completely transformed into other compounds by $\mathrm{AgNO}_{3}$ and these compounds have different $\mathrm{R}_{\mathrm{f}}$ values with 6 and 7, we can determine whether starting material 6 was present after spraying phosphomolybdic acid solution $(5 \%$ in EtOH) on TLC plate. By this simple technique, WohlZiegler bromination can been quenched appropriately and intermediate 7 was obtained in $82 \%$ yields. The total yield from $L$-alanine to 7 was $51.8 \%$.

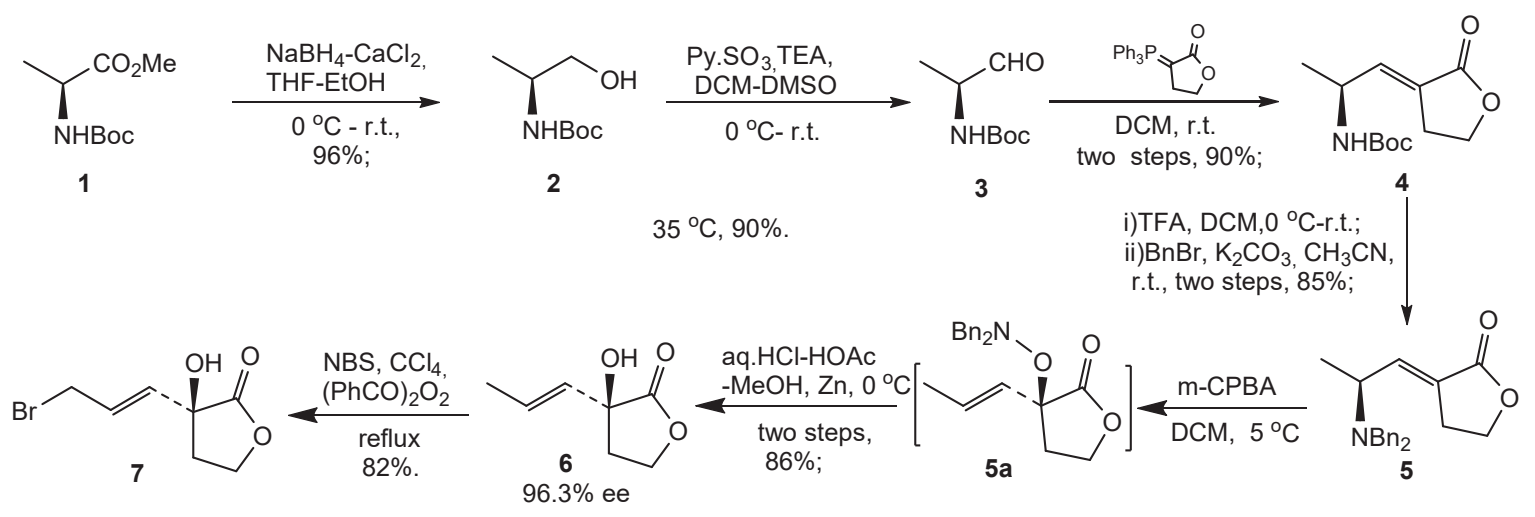

Scheme 1. Synthesis of key intermediate 7.

With bromide 7 in hand, Barbier-type coupling with acetone was investigated (Scheme 2). A variety of coupling reagents $\left(\mathrm{Zn}, \mathrm{Mg}, \mathrm{Sn}, \mathrm{Al}^{25}\right.$ with additives such as saturated $\mathrm{NH}_{4} \mathrm{Cl}$, ${ }^{26}$ saturated $\mathrm{NH}_{4} \mathrm{OAc},{ }^{27} \mathrm{DMF}^{28}$ and formamide ${ }^{29}$ ) were examined and none lead to satisfactory 
result. With THF, THF- $\mathrm{H}_{2} \mathrm{O}$ or saturated $\mathrm{NH}_{4} \mathrm{Cl}-\mathrm{H}_{2} \mathrm{O}$ as solvents, Barbier-type coupling was very sluggish, giving only a trace of $\mathbf{8}$ after $12 \mathrm{~h}$ and the main byproduct was allyl-metal protonolysis product 6 . With $\mathrm{DMF}$ as solvents and solid $\mathrm{NH}_{4} \mathrm{Cl}$ as additives, ${ }^{30}$ compound 7 was transformed into 8 in $60 \%$ yield, together with dehydroxylation material 9 in $20 \%$ yield. Generally, excessive bromide ought to be employed for increasing the yield, in our present work, however, acetone was used in two-fold excess. Further increasing loading of acetone will decrease the isolated yield. Next, $(\boldsymbol{R})-\mathbf{2 0}$ was smoothly obtained from $\mathbf{8}$ via the sequential step of catalytic hydrogenation and lactone oxidation. The total yield from $\mathbf{7}$ to $(\boldsymbol{R})-\mathbf{2 0}$ was $51.3 \%$.

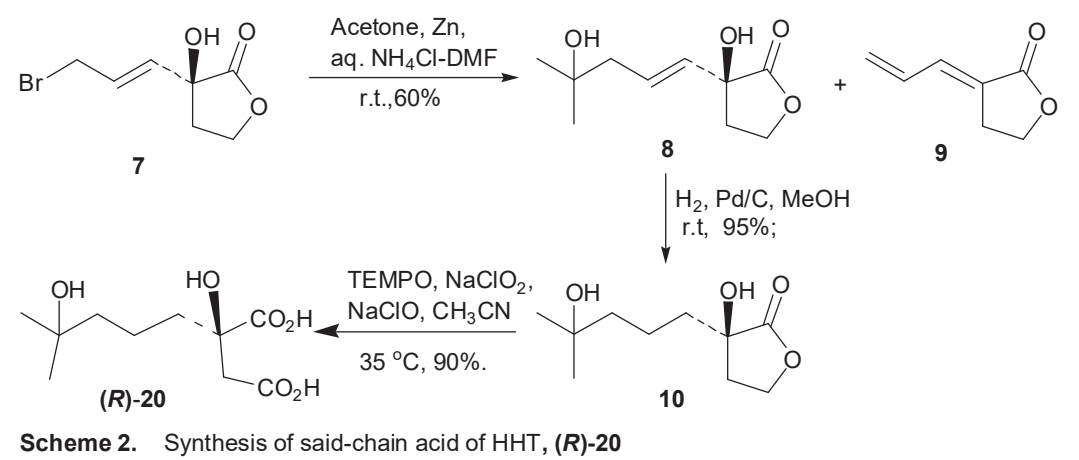

Direct oxidation of $\mathbf{6}$ using $\mathrm{SeO}_{2}{ }^{31}$ to obtain enal 11 was proved to lead to decomposed products, the easily available intermediate 7 was inevitably employed to prepare unstable $\mathbf{1 1}$ by IBX oxidation ${ }^{32}$, which is the first step for the elaboration of side-chain acid of HT, $(\boldsymbol{R})$-30 (Scheme 3). The addition of MeMgBr to enal 11 produced 12, ${ }^{33}$ further oxidation of 15 with IBX furnished enone $13 .{ }^{34}$ Subsequent $\mathrm{MeMgBr}$ addition to enone $13,{ }^{33}$ double bond hydrogenation and TEMPO-mediated lactone oxidation furnished $(\boldsymbol{R})-\mathbf{3 0}$ in $71.8 \%$ yield over three steps. ${ }^{9}$ The total yield from 7 to $(\boldsymbol{R})-\mathbf{3 0}$ was $52.2 \%$.

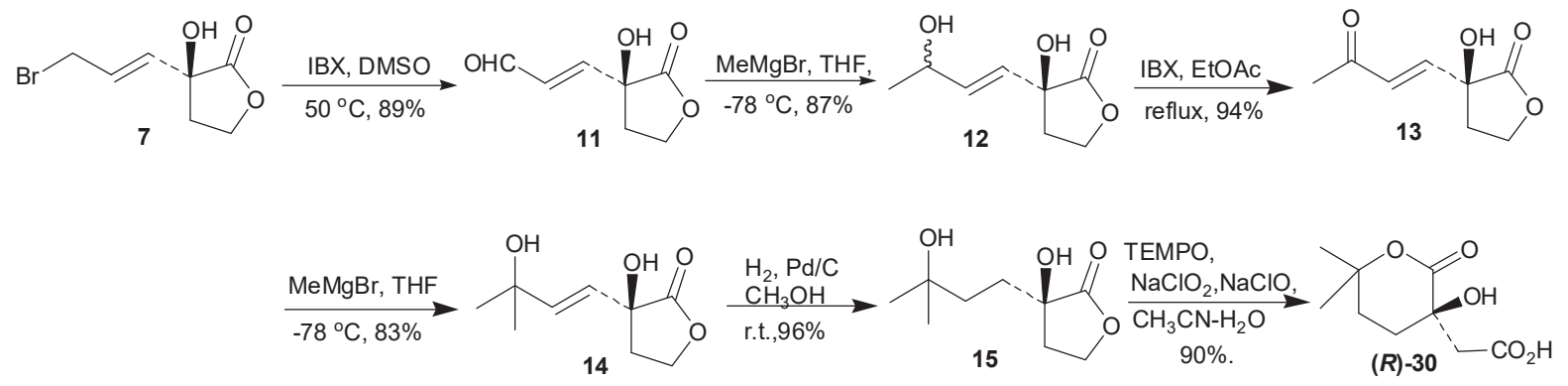

Scheme 3. Synthesis of said-chain acid of $\mathrm{HT},(R)-30$.

\section{Conclusions}

The common used intermediate 7 for the synthesis of side-chain acids of HHT and HT was conveniently prepared from $L$-alanine in $51.8 \%$ yield over eight steps with more than $96 \%$ ee . 
Meanwhile, Parikh-Doering oxidization was confirmed to be suitable for the oxidation of $N$-Boc$L$-Ala-OH into $N$-Boc- $L$-Alaninal with no racemization. The introduced technique for monitoring Wohl-Ziegler bromination by TLC could be widely used. In the respect of conciseness and yield, this second-generation route is superior to the reported one (overall yield: $26.6 \%$ vs $25 \%$ for $(\boldsymbol{R})$ $\mathbf{2 0}, 27.0 \%$ vs $18 \%$ for $(\boldsymbol{R})-\mathbf{3 0})$. In addition, the key intermediate 7 was a versatile synthon, which can couple with isopropyl bromide to produce deoxyhomoharringtonine skeleton or couple with 2,2-dimethyloxirane to produce bishomoharringtonine skeleton ${ }^{35}$.

\section{Experimental Section}

General. For product purification by flash column chromatography, silica gel (200 300 mesh) and petroleum ether (b.p. $60 \sim 90{ }^{\circ} \mathrm{C}$ ) are used. All solvents were purified and dried by standard techniques, and distilled prior to use. All organic extracts were dried over $\mathrm{MgSO}_{4}$, unless otherwise noted. IR spectra were recorded using $\mathrm{KBr}$ disks in the $400-4000 \mathrm{~cm}^{-1}$ region. Optical rotations were measured using a polarimeter. HR-MS was obtained by ESI (positive ion mode) on TOF mass analyzer. Melting points were determined without correction. ${ }^{1} \mathrm{H}$ and ${ }^{13} \mathrm{C}$ NMR spectra were recorded with TMS as an internal standard and $\mathrm{CDCl}_{3}$ or $\mathrm{CD}_{3} \mathrm{COCD}_{3}$ as solvent. The enantiomeric excess (e.e.) of the products were determined by HPLC using a Chiralpak IC $(250 \mathrm{~mm} \times 4.6 \mathrm{~mm}, 5 \mu \mathrm{m})$ and $\mathrm{UV}$ detector at $25^{\circ} \mathrm{C}$ and detected at $205 \mathrm{~nm}$. The mobile phase used was n-hexane - isopropanol (70:30) at a flow rate of $0.8 \mathrm{~mL} \cdot \mathrm{min}^{-1}$.

((S)-2-Hydroxy-1-methylethyl)carbamic acid tert-butyl ester (2). To a solution of 1 (5 g, $24.606 \mathrm{mmol})$ in THF/ethanol $(12 \mathrm{~mL} / 22 \mathrm{~mL})$ was added $\mathrm{CaCl}_{2}(5.462 \mathrm{~g}, 49.212 \mathrm{mmol})$ and $\mathrm{NaBH}_{4}(3.724 \mathrm{~g}, 98.425 \mathrm{mmol})$ successively at $0{ }^{\circ} \mathrm{C}$. The mixture was allowed to reach room temperature and stirred for $6 \mathrm{~h}$. Then the solution was poured into citric acid/ice $(80 \mathrm{~mL})$ and extracted with EtOAc $(3 \times 100 \mathrm{~mL})$. The combined extracts were washed with brine, dried and concentrated under reduced pressure. The residue was purified by column chromatography (petroleum ether/EtOAc, 2.5:1) to afford $2(4.311 \mathrm{~g}, 96 \%)$ as a colourless crystalline solid. mp $59.5-60.5{ }^{\circ} \mathrm{C}$ (petroleum ether - EtOAc); $[\alpha]^{20} \mathrm{D}-11.4\left(\mathrm{c} 0.99, \mathrm{CHCl}_{3}\right)$.

(S, E)-tert-Butyl(1-(2-oxodihydrofuran-3(2H)-ylidene)propan-2-yl)carbamate (4). To a solution of $2(1.500 \mathrm{~g}, 8.560 \mathrm{mmol})$ and TEA $(3.58 \mathrm{~mL}, 25.680 \mathrm{mmol})$ in dry $\mathrm{CH}_{2} \mathrm{Cl}_{2}(12 \mathrm{~mL})$ was added a solution of Py. $\mathrm{SO}_{3}(4.087 \mathrm{~g}, 25.680 \mathrm{mmol})$ in dry DMSO $(12 \mathrm{~mL})$ at $0{ }^{\circ} \mathrm{C}$. Then the reaction mixture was allowed to reach room temperature and stirred for $35 \mathrm{~min}$, quenched with water/ice $(30 \mathrm{~mL})$. The solution was extracted with $\mathrm{CH}_{2} \mathrm{Cl}_{2}(3 \times 60 \mathrm{~mL})$ and washed successively with $10 \%$ citric acid $(2 \times 20 \mathrm{~mL}), \mathrm{H}_{2} \mathrm{O}(2 \times 20 \mathrm{~mL})$, saturated $\mathrm{NaHCO}_{3}(20 \mathrm{~mL})$, brine $(20 \mathrm{~mL})$, dried over $\mathrm{MgSO}_{4}$, and concentrated under reduced pressure to afford crude $\mathbf{3}$ as a white solid, which was used immediately for the next step without further purification. $[\alpha]^{20} \mathrm{D}$ $-28.0\left(\mathrm{c} 0.72, \mathrm{CHCl}_{3}\right)$; IR (KBr) 1526, 1696, 2814, 2938, 2981, $3331 \mathrm{~cm}^{-1}$. 
To a solution of crude aldehyde 3 in dry $\mathrm{CH}_{2} \mathrm{Cl}_{2}(10 \mathrm{~mL})$ was added 1-butyrolactonylidene triphenylphosphorane $(3.344 \mathrm{~g}, 9.654 \mathrm{mmol})$. The mixture was stirred at room temperature for 1 $\mathrm{h}$. Then solvent was removed under reduced pressure and the residue was purified by chromatography (petroleum ether/ EtOAc, 3:1) to yield olefin $4(1.859 \mathrm{~g}, 90 \%, 99 \%$ ee) as a white crystal: $\mathrm{mp} 101.5-103.0{ }^{\circ} \mathrm{C}$ (petroleum ether-EtOAc); IR $(\mathrm{KBr}) 3397,1752 \mathrm{~cm}^{-1} ;[\alpha]^{20} \mathrm{D}$ $-28.0\left(\mathrm{c} 0.72, \mathrm{CHCl}_{3}\right) ; 1 \mathrm{H} \mathrm{NMR}\left(400 \mathrm{MHz}, \mathrm{CDCl}_{3}\right) \delta 6.43(\mathrm{~d}, J 8.4 \mathrm{~Hz}, 1 \mathrm{H}), 4.69(\mathrm{~d}, J 5.6 \mathrm{~Hz}$, $1 \mathrm{H}), 4.34-4.30(\mathrm{~m}, 3 \mathrm{H}), 3.10(\mathrm{~m}, 1 \mathrm{H}), 2.88-2.80(\mathrm{~m}, 1 \mathrm{H}), 1.34(\mathrm{~s}, 9 \mathrm{H}), 1.21(\mathrm{~d}, J 7.2,1 \mathrm{H}) ;{ }^{13} \mathrm{C}$ NMR (100 MHz, $\left.\mathrm{CDCl}_{3}\right) \delta 171.5(\mathrm{C}), 155.0(\mathrm{C}), 140.62(\mathrm{CH}), 125.7(\mathrm{C}), 79.8(\mathrm{C}), 65.7\left(\mathrm{CH}_{2}\right)$, $46.0(\mathrm{CH}), 28.4\left(3 \mathrm{CH}_{3}\right), 24.9\left(\mathrm{CH}_{2}\right), 20.0\left(\mathrm{CH}_{3}\right)$; HRMS (ESI-TOF) $\mathrm{m} / z$ : $[\mathrm{M}+\mathrm{Na}]^{+}$calcd for $\mathrm{C}_{12} \mathrm{H}_{19} \mathrm{NO}_{4} \mathrm{Na}$, 264.1212; found 264.1213;

(S, E)-3-(2-(Dibenzylamino)propylidene)dihydrofuran-2(3H)-one (5). To a solution of $N$-Boc olefin $4(3.2 \mathrm{~g}, 13.263 \mathrm{mmol})$ in dry $\mathrm{CH}_{2} \mathrm{Cl}_{2}(20 \mathrm{~mL})$ was added trifluoroacetic acid (7.561 g, $66.313 \mathrm{mmol}$ ), and the reaction was refluxed for $3 \mathrm{~h}$. Then, it was evaporated to give a white solid. To a solution of the white solid in dry acetonitrile $(40 \mathrm{~mL})$ was added $\mathrm{K}_{2} \mathrm{CO}_{3}(5.499 \mathrm{~g}$, $39.795 \mathrm{mmol})$, followed by $\mathrm{BnBr}(4.991 \mathrm{~g}, 29.183 \mathrm{mmol})$. The reaction mixture was stirred at reflux for $3 \mathrm{~h}$, at which point most of the solvent was evaporated under reduced pressure. The residue was diluted with water, and extracted with EtOAc $(3 \times 60 \mathrm{~mL})$. The combined extracts were washed with brine, dried, and concentrated under reduced pressure. The residue was recrystallized from petroleum ether and EtOAc to give $N, N$-dibenzyl olefin 5 (3.624 g, 85\%, $99 \%$ ee ) as white crystals: $\mathrm{mp} 101.5-103.0{ }^{\circ} \mathrm{C}$; $\mathrm{IR}(\mathrm{KBr}) 3056,3028,1751 \mathrm{~cm}^{-1} ;[\alpha]^{20} \mathrm{D}+3.6(\mathrm{c}$ 1.0, $\left.\mathrm{CHCl}_{3}\right) ;{ }^{1} \mathrm{H}$ NMR $\left(400 \mathrm{MHz}, \mathrm{CDCl}_{3}\right) \delta 7.39-7.33(\mathrm{~m}, 8 \mathrm{H}), 7.29-7.24(\mathrm{~m}, 2 \mathrm{H}), 6.91$ (dt, $J$ $6.0,9.6,1 \mathrm{H}), 4.36-4.31(\mathrm{~m}, 2 \mathrm{H}), 3.82(\mathrm{~d}, J 13.6 \mathrm{~Hz}, 2 \mathrm{H}), 3.56-3.52(\mathrm{~m}, 3 \mathrm{H}), 2.63-2.59(\mathrm{~m}, 2 \mathrm{H})$, $1.63(\mathrm{~s}, 1 \mathrm{H}), 1.30(\mathrm{~d}, J 6.8 \mathrm{~Hz}, 3 \mathrm{H}) ;{ }^{13} \mathrm{C} \mathrm{NMR}\left(100 \mathrm{MHz}, \mathrm{CDCl}_{3}\right) \delta 171.1(\mathrm{C}), 140.7(\mathrm{C}), 139.8$ $(\mathrm{CH}), 128.5(\mathrm{CH}), 128.3(\mathrm{CH}), 127.0(\mathrm{CH}), 126.7(\mathrm{C}), 65.5\left(\mathrm{CH}_{2}\right), 54.0\left(\mathrm{CH}_{2}\right), 53.0(\mathrm{CH}), 25.1$ $\left.\mathrm{CH}_{2}\right), 16.3\left(\mathrm{CH}_{3}\right)$; HRMS (ESI-TOF) $m / z$ : $[\mathrm{M}+\mathrm{Na}]^{+}$calcd for $\mathrm{C}_{21} \mathrm{H}_{23} \mathrm{NO}_{2} \mathrm{Na}, 344.1626$; found 344.1628 .

(S, E)-3-Hydroxy-3-(prop-1-en-1-yl) dihydrofuran-2(3H)-one (6). To a solution of 5 (500 mg, $1.556 \mathrm{mmol})$ in $\mathrm{CH}_{2} \mathrm{Cl}_{2}(6 \mathrm{~mL})$ was added $m$-CPBA $(80 \%$ pure, $369 \mathrm{mg}, 1.711 \mathrm{mmol})$ at $5{ }^{\circ} \mathrm{C}$. The reaction mixture was stirred at this temperature for $10 \mathrm{~min}$. After the suspension was filtered, the filtrate was washed with $10 \% \mathrm{NaOH}(3 \times 5 \mathrm{~mL})$, saturated $\mathrm{NaHCO}_{3}(2 \times 10 \mathrm{~mL})$, and saturated $\mathrm{NaCl}(2 \times 10 \mathrm{~mL})$, dried over $\mathrm{MgSO}_{4}$, filtered, and concentrated at $40{ }^{\circ} \mathrm{C}$ under reduced pressure to give an oily residue (crude 5a), which was used in the next step without further purification. To a solution of crude 5a $(1.556 \mathrm{mmol})$ in $\mathrm{MeOH}(5 \mathrm{~mL})$ was added glacial acetic acid $(0.6 \mathrm{~mL}, 10.892 \mathrm{mmol})$, hydrochloric acid $(0.9 \mathrm{~mL}, 10.892 \mathrm{mmol}, 37 \% \mathrm{HCl})$ and zinc powder $(1.017 \mathrm{~g}, 15.56 \mathrm{mmol})$ at $0{ }^{\circ} \mathrm{C}$. The reaction mixture was stirred at this temperature for $10 \mathrm{~min}$. Then the suspension was filtered and washed with EtOAc, and the filtrate was neutralized with $25 \%$ ammonium hydroxide solution to $\mathrm{pH} 8$. The mixture was extracted with EtOAc $(3 \times 30 \mathrm{~mL})$. The combined extracts were washed with brine, dried, concentrated. The residue was purified by chromatography (petroleum ether/EtOAc, 2:1) to afford $6(190 \mathrm{mg}, 86 \%$, $96.3 \%$ ee) as a colorless oil: IR $(\mathrm{KBr}) 3445,1770 \mathrm{~cm}^{-1}, 1667 \mathrm{~cm}^{-1} ;[\alpha]^{20} \mathrm{D}+74.5\left(\mathrm{c} 1.2, \mathrm{CHCl}_{3}\right)$; 
${ }^{1} \mathrm{H}$ NMR $\left(400 \mathrm{MHz}, \mathrm{CDCl}_{3}\right) \delta 5.86(\mathrm{dq}, J 15.7,6.5 \mathrm{~Hz}, 1 \mathrm{H}), 5.63(\mathrm{~d}, J 15.7,1.2 \mathrm{~Hz}, 1 \mathrm{H}), 4.39$ (td, $J 8.8,3.0 \mathrm{~Hz}, 1 \mathrm{H}), 4.19-4.13(\mathrm{~m}, 1 \mathrm{H}), 3.35(\mathrm{brs}, 1 \mathrm{H}), 2.51-2.42(\mathrm{~m}, 1 \mathrm{H}), 2.40-2.34(\mathrm{~m}, 1 \mathrm{H})$, 1.76(dd, $J 6.5,1.0 \mathrm{~Hz}, 3 \mathrm{H}) ;{ }^{13} \mathrm{C} \mathrm{NMR}\left(100 \mathrm{MHz}, \mathrm{CDCl}_{3}\right) \delta 177.9(\mathrm{C}), 129.0(\mathrm{CH}), 128.8(\mathrm{CH})$, $75.0(\mathrm{C}), 65.0\left(\mathrm{CH}_{2}\right), 36.2\left(\mathrm{CH}_{2}\right), 17.8\left(\mathrm{CH}_{3}\right)$; HRMS (ESI-TOF) $\mathrm{m} / \mathrm{z}:[\mathrm{M}+\mathrm{Na}]^{+}$calcd for $\mathrm{C}_{7} \mathrm{H}_{10} \mathrm{O}_{3} \mathrm{Na}, 165.0528$; found 165.0526 .

(S, E)-3-(3-Bromoprop-1-en-1-yl)-3-hydroxydihydrofuran-2(3H)-one (7). To a solution of 6 $(1.2 \mathrm{~g}, 8.442 \mathrm{mmol})$ in dry $\mathrm{CCl}_{4}(12 \mathrm{~mL})$ was added $N$-bromosuccinimide $(1.803 \mathrm{~g}, 10.110 \mathrm{mmol})$ and benzoyl peroxide $(102 \mathrm{mg}, 0.442 \mathrm{mmol})$. The reaction was stirred under reflux and monitored by TLC using the method described in the text. After the reaction was complete, the suspension was filtered and the filtrate was concentrated under reduced pressure. The residue was purified by chromatography (petroleum ether/EtOAc, 3: 1) to give $7(1.530 \mathrm{mg}, 82 \%)$ as pale-yellow sticky oil. $[\alpha]^{20}{ }_{\mathrm{D}}+46.0\left(\mathrm{c} 1.06, \mathrm{CHCl}_{3}\right)$; IR (KBr) 3426, $1770 \mathrm{~cm}^{-1} ;{ }^{1} \mathrm{H}$ NMR (400 $\left.\mathrm{MHz}, \mathrm{CDCl}_{3}\right) \delta 6.12-6.05(\mathrm{~m}, 1 \mathrm{H}), 5.90(\mathrm{~d}, J 15.5 \mathrm{~Hz}, 1 \mathrm{H}), 4.47-4.42(\mathrm{~m}, 1 \mathrm{H}), 4.23(\mathrm{td}, J 9.0$, $6.6 \mathrm{~Hz}, 1 \mathrm{H}), 3.96(\mathrm{dd}, J 7.2,0.9 \mathrm{~Hz}, 2 \mathrm{H}), 3.07(\mathrm{~s}, 1 \mathrm{H}), 2.55-2.48(\mathrm{~m}, 1 \mathrm{H}), 2.42$ (ddd, $J$ 13.1, 6.5, $3.7 \mathrm{~Hz}, 1 \mathrm{H}) ;{ }^{13} \mathrm{C} \mathrm{NMR}\left(100 \mathrm{MHz}, \mathrm{CDCl}_{3}\right) \delta 176.9(\mathrm{C}), 131.9(\mathrm{CH}), 129.1(\mathrm{CH}), 74.6(\mathrm{C})$, $65.1\left(\mathrm{CH}_{2}\right), 36.1\left(\mathrm{CH}_{2}\right), 30.5\left(\mathrm{CH}_{2}\right)$; HRMS (ESI-TOF) $\mathrm{m} / z$ : $[\mathrm{M}+\mathrm{Na}]^{+}$calcd for $\mathrm{C}_{7} \mathrm{H}_{9} \mathrm{BrO}_{3} \mathrm{Na}$, 242.9633; found 242.9634 .

( $S, E)-3-H y d r o x y-3-(4-h y d r o x y-4-m e t h y l p e n t-1-e n-1-y l)$ dihydrofuran-2(3H)-one (8). To a solution of bromide 7 (685 mg, $3.099 \mathrm{mmol})$ and acetone $(540 \mathrm{mg}, 9.297 \mathrm{mmol})$ in DMF (5 mL) was added zinc dust (304 mg, $4.649 \mathrm{mmol})$ and $\mathrm{NH}_{4} \mathrm{Cl}(166 \mathrm{mg}, 3.099 \mathrm{mmol})$ in one portion at room temperature. The reaction mixture was stirred at this temperature for $1.5 \mathrm{~h}$, then extracted with EtOAc $(3 \times 40 \mathrm{~mL})$, washed with saturated brine $(2 \times 10 \mathrm{~mL})$, dried and concentrated. The residue was purified by chromatography (petroleum ether/EtOAc, 1:3) to afford diol 8 (373 mg, $60 \%)$ as a colorless oil and conjugated olefin $9(77 \mathrm{mg}, 20 \%)$ as a colorless oil. Diol 8: $[\alpha]^{25} \mathrm{D}^{+}$ 30.1 (c 0.98, EtOH); IR (KBr) :3419, $1770 \mathrm{~cm}^{-1} ;{ }^{1} \mathrm{H}$ NMR $\left(400 \mathrm{MHz}, \mathrm{CDCl}_{3}\right) \delta 5.97-5.89$ (m, $1 \mathrm{H}), 5.69$ (d, $J 15.7 \mathrm{~Hz}, 1 \mathrm{H}), 4.59$ (brs, $1 \mathrm{H}), 4.41$ (td, $J 8.3,3.5 \mathrm{~Hz}, 1 \mathrm{H}), 4.22-4.16(\mathrm{~m}, 1 \mathrm{H}), 2.85$ (brs, 1H), 2.51-2.43 (m, 1H), 2.40-2.35 (m, 1H), 2.24 (d, J $7.5 \mathrm{~Hz}, 2 \mathrm{H}), 1.23$ (d, J 5.2 Hz, 6H); ${ }^{13} \mathrm{C}$ NMR (100 MHz, $\left.\mathrm{CDCl}_{3}\right) \delta 178.1(\mathrm{C}), 131.4(\mathrm{CH}), 129.5(\mathrm{CH}), 75.1(\mathrm{C}), 70.9(\mathrm{C}), 65.2$ $\left(\mathrm{CH}_{2}\right), 46.1\left(\mathrm{CH}_{2}\right), 36.4\left(\mathrm{CH}_{2}\right), 29.3\left(\mathrm{CH}_{3}\right), 29.0\left(\mathrm{CH}_{3}\right)$; HRMS (ESI-TOF) $m / z$ : $[\mathrm{M}+\mathrm{Na}]^{+}$calcd for $\mathrm{C}_{10} \mathrm{H}_{16} \mathrm{O}_{4} \mathrm{Na}$, 223.0946; found 223.0948; conjugated olefin 9: IR (KBr): 3446, 1748, 1656 $\mathrm{cm}^{-1} ;{ }^{1} \mathrm{H} \mathrm{NMR}\left(400 \mathrm{MHz}, \mathrm{CDCl}_{3}\right) \delta 7.10(\mathrm{~d}, J 11.4 \mathrm{~Hz}, 1 \mathrm{H}), 6.46(\mathrm{dt}, J 16.9,10.8 \mathrm{~Hz}, 1 \mathrm{H}), 5.66$ $(\mathrm{dd}, J 16.9,10.0 \mathrm{~Hz}, 2 \mathrm{H}), 4.42(\mathrm{t}, J 7.4 \mathrm{~Hz}, 2 \mathrm{H}), 3.01(\mathrm{td}, J 7.3,2.2 \mathrm{~Hz}, 2 \mathrm{H}) ;{ }^{13} \mathrm{C}$ NMR $(100$ $\left.\mathrm{MHz}, \mathrm{CDCl}_{3}\right) \delta 171.6(\mathrm{C}), 135.8(\mathrm{CH}), 132.2(\mathrm{CH}), 126.7\left(\mathrm{CH}_{2}\right), 125.3(\mathrm{C}), 65.4\left(\mathrm{CH}_{2}\right), 25.3$ $\left(\mathrm{CH}_{2}\right)$; HRMS (ESI-TOF) $\mathrm{m} / z$ : $[\mathrm{M}+\mathrm{Na}]^{+}$calcd for $\mathrm{C}_{7} \mathrm{H}_{8} \mathrm{O}_{2} \mathrm{Na}, 147.0422$; found 147.0420.

(R)-3-Hydroxy-3-(4-hydroxy-4-methylpentyl) dihydrofuran-2(3H)-one (10). To a solution of $8(516 \mathrm{mg}, 2.577 \mathrm{mmol})$ in $\mathrm{MeOH}(6 \mathrm{~mL})$ was added $10 \% \mathrm{Pd} / \mathrm{C}(52 \mathrm{mg})$. The mixture was stirred under hydrogen atmosphere (60 psi) at room temperature for $2 \mathrm{~h}$. Then catalyst was removed by filtration over Celite and washed with $\mathrm{MeOH}(3 \times 5 \mathrm{~mL})$. The filtrate was concentrated under reduced pressure and the residue was purified by chromatography (petroleum ether/EtOAc, 1:2.5) to afford $10(495 \mathrm{mg}, 95 \%)$ as a white solid. $[\alpha]^{25} \mathrm{D}+14.4(\mathrm{c} 0.8, \mathrm{EtOH}) ; \mathrm{mp}$ 
97.8-98.9 ${ }^{\circ} \mathrm{C}$ (EtOAc); IR (KBr): 3500, 3280, $1769 \mathrm{~cm}^{-1} ;{ }^{1} \mathrm{H}$ NMR $\left(400 \mathrm{MHz}, \mathrm{CDCl}_{3}\right) \delta$ 4.42-4.37 (m, 1H), 4.26-4.20 (m, 1H), 3.72-3.67 (m, 1H), 2.54 (brs, 1H), 2.38-2.25 (m, 2H), 1.83-1.78 (m, 1H), 1.67-1.60 (m, 2H), 1.52-1.40 (m, 3H), $1.21(\mathrm{~s}, 6 \mathrm{H}) ;{ }^{13} \mathrm{C}$ NMR $(100 \mathrm{MHz}$, $\left.\mathrm{CDCl}_{3}\right) \delta 179.2(\mathrm{C}), \quad 74.7(\mathrm{C}), \quad 71.1(\mathrm{C}), 65.5\left(\mathrm{CH}_{2}\right), \quad 43.4\left(\mathrm{CH}_{2}\right), 36.7\left(\mathrm{CH}_{2}\right), 34.7\left(\mathrm{CH}_{2}\right)$, $29.3\left(\mathrm{CH}_{3}\right), 29.2\left(\mathrm{CH}_{3}\right), 17.9\left(\mathrm{CH}_{2}\right)$; HRMS (ESI-TOF) $\mathrm{m} / z$ : $[\mathrm{M}+\mathrm{Na}]^{+}$calcd for $\mathrm{C}_{10} \mathrm{H}_{18} \mathrm{O}_{4} \mathrm{Na}$ 225.1103 , found 225.1103 .

(R)-2-Hydroxy-2-(4-hydroxy-4-methylpentyl)succinic acid (20). Saturated diol 10 (857 mg, $4.237 \mathrm{mmol})$ was stirred for $20 \mathrm{~min}$ with $5 \mathrm{M} \mathrm{NaOH}(1.6 \mathrm{~mL})$. Sodium phosphate buffer $(18 \mathrm{ml}$, $0.67 \mathrm{M})$ was added and the $\mathrm{pH}$ was adjusted to $6.8(5 \mathrm{M} \mathrm{HCl})$. Then, acetonitrile $(16.5 \mathrm{ml})$ and TEMPO (232 mg, $1.483 \mathrm{mmol})$ were added and the mixture was heated to $35^{\circ} \mathrm{C}$. Over a period of $3 \mathrm{~h}$ sodium hypochlorite solution $\left(5.38 \mathrm{~mL}\right.$ in $\left.29.82 \mathrm{~mL} \mathrm{H} \mathrm{H}_{2} \mathrm{O}, 0.24 \mathrm{M}\right)$ and $\mathrm{NaClO}_{2}(978 \mathrm{mg}$, $80 \%, 8.65 \mathrm{mmol}$ in $4.24 \mathrm{~mL} \mathrm{H}_{2} \mathrm{O}$ ) were added dropwise from separate syringes at $35{ }^{\circ} \mathrm{C}$ while stirring (Caution: do not mix sodium hypochlorite solution and $\mathrm{NaClO}_{2}$ before adding to the reaction!). At room temperature, the $\mathrm{pH}$ was adjusted to 8.5 with $5 \mathrm{M} \mathrm{NaOH}$. The mixture was quenched with $\mathrm{Na}_{2} \mathrm{SO}_{3}\left(9.92 \mathrm{~mL}, 1.923 \mathrm{~g}\right.$ in $15 \mathrm{~mL}$ water) at $0{ }^{\circ} \mathrm{C}$ and the resulting solution was stirred at room temperature for $1 \mathrm{~h}(\mathrm{pH} \mathrm{8.5-9.0).} \mathrm{After} \mathrm{acidification} \mathrm{to} \mathrm{pH} \mathrm{1,} \mathrm{most} \mathrm{of} \mathrm{the} \mathrm{water}$ was removed using rotary evaporator. Then the mixture was extracted with EtOAc $(6 \times 30 \mathrm{~mL})$, washed with saturated brine $(2 \times 10 \mathrm{~mL})$, dried over $\mathrm{MgSO}_{4}$ and concentrated under reduced pressure. The residue was purified by chromatography (petroleum ether /EtOAc, 1:6) to afford 20 (893 mg, 90\%) as a white crystalline solid. $[\alpha]^{25}{ }_{\mathrm{D}}-10.2$ (c 1.08, MeOH); mp $112.1-112.9^{\circ} \mathrm{C}$ (EtOAc); IR (KBr) 3569, 3456, $1709 \mathrm{~cm}^{-1} ;{ }^{1} \mathrm{H}$ NMR (400 MHz, d6-acetone) $\delta 2.95$ (d, $J 16.3 \mathrm{~Hz}$, $1 \mathrm{H}), 2.67(\mathrm{~d}, J 16.3 \mathrm{~Hz}, 1 \mathrm{H}), 1.76-1.55(\mathrm{~m}, 3 \mathrm{H}), 1.45$ (dd, $J 9.3,6.4 \mathrm{~Hz}, 2 \mathrm{H}), 1.39-1.29$ (m, 1H), 1.17 (s, 6H); ${ }^{13} \mathrm{C}$ NMR (100MHz, d6-acetone) $\delta 175.8$ (C), 171.7 (C), 74.7 (C), $69.5(\mathrm{C})$, $43.8\left(\mathrm{CH}_{2}\right), 42.9\left(\mathrm{CH}_{2}\right), 39.9\left(\mathrm{CH}_{2}\right), 29.0\left(\mathrm{CH}_{3}\right), 28.8\left(\mathrm{CH}_{3}\right), 18.0\left(\mathrm{CH}_{2}\right)$; HRMS (ESI -TOF) $m / z$ : calcd for $[\mathrm{M}+\mathrm{Na}-2 \mathrm{H}]^{+}, 255.0845$, found 255.0847 .

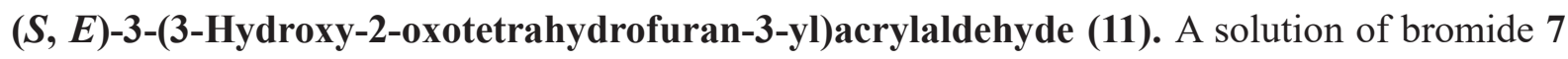
(609 $\mathrm{mg}, 2.755 \mathrm{mmol})$ in DMSO $(8 \mathrm{~mL})$ was added IBX $(1.543 \mathrm{~g}, 5.510 \mathrm{mmol})$. The reaction mixture was stirred at $50{ }^{\circ} \mathrm{C}$ for $1.5 \mathrm{~h}$. Then the mixture was diluted with EtOAc $(100 \mathrm{~mL})$, washed with saturated aqueous $\mathrm{NaHCO}_{3}(2 \times 10 \mathrm{~mL})$, brine $(10 \mathrm{~mL})$, dried over $\mathrm{MgSO}_{4}$ and concentrated under reduced pressure. The residue was purified by chromatography (petroleum ether /EtOAc, 1:1) to afford 11 (383 mg, 89\%) as a colorless oil. IR (KBr) 3420, 2923, 2850, 1771, $1690 \mathrm{~cm}^{-1}$; ${ }^{1} \mathrm{H}$ NMR $\left(400 \mathrm{MHz}, \mathrm{CDCl}_{3}\right) \delta 9.63(\mathrm{~d}, J 7.5 \mathrm{~Hz}, 1 \mathrm{H}), 6.85(\mathrm{~d}, J 15.8 \mathrm{~Hz}, 1 \mathrm{H})$, $6.46(\mathrm{dd}, J 15.8,7.5 \mathrm{~Hz}, 1 \mathrm{H}), 4.56-4.52(\mathrm{~m}, 1 \mathrm{H}), 4.40-4.35(\mathrm{~m}, 1 \mathrm{H}), 4.09(\mathrm{~s}, 1 \mathrm{H}), 2.63-2.50(\mathrm{~m}$, 2H); ${ }^{13} \mathrm{C}$ NMR (100 MHz, $\left.\mathrm{CDCl}_{3}\right) \delta 192.8(\mathrm{CH}), 175.9(\mathrm{C}), 151.3(\mathrm{CH}), 132.3(\mathrm{CH}), 75.2(\mathrm{C})$, $65.8\left(\mathrm{CH}_{2}\right), 36.2\left(\mathrm{CH}_{2}\right)$; HRMS (ESI-TOF) $\mathrm{m} / z$ : $[\mathrm{M}+\mathrm{Na}]^{+}$calcd for $\mathrm{C}_{7} \mathrm{H}_{8} \mathrm{O}_{4} \mathrm{Na}, 179.0320$, found 179.0322 .

(3S)-3-Hydroxy-3-((E)-3-hydroxybut-1-en-1-yl) dihydrofuran-2(3H)-one (12). A solution of methylmagnesium bromide in ether $(4 \mathrm{~mL}, 4.6 \mathrm{mmol})$ was added to a solution of $11(653 \mathrm{mg}$, $4.182 \mathrm{mmol})$ in THF $(10 \mathrm{ml})$ at $-78{ }^{\circ} \mathrm{C}$. The reaction mixture was allowed to reach room temperature. After further stirred at room temperature for $30 \mathrm{~min}$, water $(10 \mathrm{ml})$ was added. The 
mixture was extracted with EtOAc $(3 \times 30 \mathrm{~mL})$. The combined extracts were washed with brine, dried, and concentrated under reduced pressure. The residue was purified by chromatography (petroleum ether/ EtOAc, 1:3) to afford 12 as colorless oil (628 mg, 87\%, dr 2:1). IR (KBr) 3405, $1767 \mathrm{~cm}^{-1} ;{ }^{1} \mathrm{H}$ NMR (400 MHz, $\left.\mathrm{CDCl}_{3}\right) \delta 5.99-5.92(\mathrm{~m}, 1 \mathrm{H}), 5.88-5.83(\mathrm{~m}, 1 \mathrm{H}), 4.45-4.39$ $(\mathrm{m}, 2 \mathrm{H}), 4.25-4.21(\mathrm{~m}, 1 \mathrm{H}), 3.63$ (brs, $1 \mathrm{H}), 3.01(\mathrm{~s}, 1 \mathrm{H}), 2.52-2.41(\mathrm{~m}, 2 \mathrm{H}), 2.40$ (brs, $1 \mathrm{H})$, 1.31 (d, J $6.4 \mathrm{~Hz}, 3 \mathrm{H}) ;{ }^{13} \mathrm{C}$ NMR (100MHz, $\left.\mathrm{CDCl}_{3}\right) 177.6$ (1C), 137.0 (1C), 136.9 (1C), 126.8 (1C), 74.8 (2C), 67.6 (2C), 65.2 (1C), 65.1 (1C), 36.2 (2C), 23.1(2C); HRMS (ESI-TOF) $\mathrm{m} / \mathrm{z}$ : [M $+\mathrm{Na}]^{+}$calcd for $\mathrm{C}_{8} \mathrm{H}_{12} \mathrm{O}_{4} \mathrm{Na}, 195.0633$; found 195.0634.

(3S)-3-Hydroxy-3-(3-oxobut-1-en-1-yl) dihydrofuran-2(3H)-one(13). To a solution of diol 12 $(520 \mathrm{mg}, 3.020 \mathrm{mmol})$ in EtOAc $(15 \mathrm{~mL})$ was added IBX $(2.537 \mathrm{~g}, 9.060 \mathrm{mmol})$ at room temperature. The reaction mixture was stirred at reflux for $3 \mathrm{~h}$. After cooling to room temperature, the insoluble material was filtered and the filtrate was concentrated under reduced pressure. The residue was purified by chromatography (petroleum ether/EtOAc, 1:1) to afford 13 (484 mg, $94 \%)$ as a colorless oil. IR(KBr): $3475,1775,1715,1677,1629 \mathrm{~cm}^{-1} ;[\alpha]^{20} \mathrm{D}=-4.1(\mathrm{c} 1.50$, $\mathrm{MeOH}) ;{ }^{1} \mathrm{H} \mathrm{NMR}\left(400 \mathrm{MHz}, \mathrm{CDCl}_{3}\right) \delta 6.79(\mathrm{~d}, J 15.9 \mathrm{~Hz}, 1 \mathrm{H}), 6.45(\mathrm{~d}, J 15.9 \mathrm{~Hz}, 1 \mathrm{H})$, 4.54-4.48 (m, 1H), 4.36-4.30 (m, 1H), 4.15-4.08 (brm, 1H), 2.59-2.46 (m, 2H), 2.31 (s, 3H); ${ }^{13} \mathrm{C} \mathrm{NMR}\left(100 \mathrm{MHz}, \mathrm{CDCl}_{3}\right) \delta 198.0,176.1,141.2,130.3,74.9,65.4,36.2,28.2$; HRMS (ESITOF) $m / z:[\mathrm{M}+\mathrm{Na}]^{+}$Calcd for $\mathrm{C}_{8} \mathrm{H}_{10} \mathrm{O}_{4} \mathrm{Na}$ 193.0477, Found 193.0478.

3-Hydroxy-3-(3-hydroxy-3-methylbut-1-en-1-yl) dihydrofuran-2(3H)-one (14). The procedure is similar to the preparation of compound 12. IR $(\mathrm{KBr}): 3421,1764,1708 \mathrm{~cm}^{-1} ;[\alpha]^{20} \mathrm{D}$ $+18.2(\mathrm{c}, 0.85, \mathrm{MeOH}) ;{ }^{1} \mathrm{H} \mathrm{NMR}\left(400 \mathrm{MHz}, \mathrm{CDCl}_{3}\right) \delta 5.99(\mathrm{~d}, J 15.82 \mathrm{~Hz}, 1 \mathrm{H}), 5.85(\mathrm{~d}, J$ $15.88 \mathrm{~Hz}, 1 \mathrm{H}), 4.46-4.41(\mathrm{~m}, 1 \mathrm{H}), 4.24-4.18(\mathrm{~m}, 1 \mathrm{H}), 2.54-2.46(\mathrm{~m}, 1 \mathrm{H}), 2.43-2.38(\mathrm{~m}, 1 \mathrm{H})$, $1.34(\mathrm{~s}, 3 \mathrm{H}) ;{ }^{13} \mathrm{C} \mathrm{NMR}\left(100 \mathrm{MHz}, \mathrm{CDCl}_{3}\right): \delta 176.5,140.7,124.3,74.8,70.6,65.0,36.3,29.8$, 29.7; HRMS (ESI-TOF) $\mathrm{m} / z$ : $[\mathrm{M}+\mathrm{Na}]^{+}$Calcd for $\mathrm{C}_{9} \mathrm{H}_{14} \mathrm{O}_{4} \mathrm{Na} 209.0790$,Found 209.0792 .

(R)-3-Hydroxy-3-(3-hydroxy-3-methylbutyl) dihydrofuran-2(3H)-one (15). The procedure is similar to the preparation of compound 10. mp 98.0-98.6 ${ }^{\circ} \mathrm{C}$ (EtOAc); IR (KBr) 3416, 3258, $1752 \mathrm{~cm}^{-1} ;[\alpha]^{20} \mathrm{D}+18.7(\mathrm{c}, 1.09, \mathrm{EtOH}) ;{ }^{1} \mathrm{H}$ NMR (400 MHz, $\left.\mathrm{CDCl}_{3}\right) \delta 4.93$ (brs, $1 \mathrm{H}$ ), 4.43-4.37 (m, 1H), 4.25-4.18 (m, 1H), 2.55 (brs, 1H), 2.44-2.35 (m, 1H), 2.28-2.20 (m, 1H), 1.98-1.83 $(\mathrm{m}, 2 \mathrm{H}), 1.70(\mathrm{t}, J 6.8 \mathrm{~Hz}, 2 \mathrm{H}), 1.28(\mathrm{~s}, 3 \mathrm{H}), 1.27(\mathrm{~s}, 3 \mathrm{H}) ;{ }^{13} \mathrm{C} \mathrm{NMR}\left(100 \mathrm{MHz}, \mathrm{CDCl}_{3}\right): \delta 179.2$, 74.2, 70.7, 65.4, 36.9, 35.8, 31.2, 30.2, 28.3; HRMS (ESI-TOF) $m / z:[\mathrm{M}+\mathrm{Na}]^{+}$Calcd for $\mathrm{C}_{9} \mathrm{H}_{16} \mathrm{O}_{4} \mathrm{Na} 211.0946$; Found 211.0947.

(R)-2-(3-Hydroxy-6, 6-dimethyl-2-oxotetrahydro-2H-pyran-3-yl) acetic acid (30). The compound 30 was obtained as a white solid, by the same procedure used for the preparation of diacid 20. mp 133.1-133.7 ${ }^{\circ} \mathrm{C}$ (EtOAc); IR (KBr) 3350 - 3000 (broad peak), 1715, $1693 \mathrm{~cm}^{-1}$; $[\alpha]^{20} \mathrm{D}+25.5(\mathrm{c}, 1.30, \mathrm{MeOH}) ;{ }^{1} \mathrm{H}$ NMR (400 MHz, d6-acetone) $\delta 1.41(\mathrm{~s}, 3 \mathrm{H}), 1.45(\mathrm{~s}, 3 \mathrm{H}), 1.78$ (dt, $J 14.0,4.28,4.00 \mathrm{~Hz}, 1 \mathrm{H}), 1.87$ (dt, $J 14.0,3.64,4.44 \mathrm{~Hz}, 1 \mathrm{H}), 2.20$ (td, $J 13.44,3.32 \mathrm{~Hz}$, 1H), $2.44(\mathrm{td}, J 13.5,3.64 \mathrm{~Hz}, 1 \mathrm{H}), 2.65(\mathrm{~d}, J 16.6 \mathrm{~Hz}, 1 \mathrm{H}), 3.10(\mathrm{~d}, J 16.56 \mathrm{~Hz}, 1 \mathrm{H}) ;{ }^{13} \mathrm{CNMR}$ (100 MHz, d6-acetone) $\delta 171.8,171.6,82.8,69.2,42.6,30.2,29.7,29.6,26.3$; HRMS (ESI-TOF) $\mathrm{m} / \mathrm{z}:[\mathrm{M}+\mathrm{Na}]^{+}$Calcd for $\mathrm{C}_{9} \mathrm{H}_{14} \mathrm{O}_{5} \mathrm{Na} 225.0739$, Found 225.0736 . 


\section{Acknowledgements}

The authors thank the National Natural Science Foundation $(21372205,21302175)$ for the financial support.

\section{References}

1. Ancliff, R. A.; Russell, A. T.; Sanderson, A. J. Chem. Commun. 2006, 3243. http://dx.doi.org/ 10.1039/b605339d

2. Berhal, F.; Tardy, S.; Pérard-Viret, J.; Royer, J. Eur. J. Org. Chem. 2009, 437. http://dx.doi.org/ 10.1002/ejoc.200800935

3. Eckelbarger, J. E.; Wilmot, J. T.; Epperson, M. T.; Thakur, C. S.; Shum, D.; Antczak, C.; Tarassishin, L.; Djaballah, H.; Gin, D. Y. Chem. Eur. J. 2008, 14, 4293. http://dx.doi.org/ 10.1002/chem.200701998

4. El Bialy, S. A. A.; Braun, H.; Tietze, L. F. Eur. J. Org. Chem. 2005, 2965. http://dx.doi.org/ 10.1002/ejoc.200500065

5. Keller, L.; Dumas, F.; d'Angelo, J. Eur. J. Org. Chem. 2003, 2488. http://dx.doi.org/ 10.1002/ejoc.200300111

6. Keller, L.; Dumas, F.; d'Angelo, J. Tetrahedron Lett. 2001, 42, 1911. http://dx.doi.org/ 10.1016/S0040-4039(01)00086-7

7. Robin, J.-P.; Dhal, R.; Dujardin, G.; Girodier, L.; Mevellec, L.; Poutot, S. Tetrahedron Lett. 1999, 40, 2931. http://dx.doi.org/ 10.1016/S0040-4039(99)00327-5

8. Hue,V. T.; Nhung, N. T. H.; Hung, M. D. Arkivoc 2014, 6, 206.

9. Yang, H.; Sun, M. R. ; Zhao, S. G.; Zhu, M.; Xie, Y.L.; Niu, C. L.; Li, C. L. J. Org. Chem. 2013, 78, 339. http://dx.doi.org/ 10.1021/j0302203g

10. Rengasamy, R.; Curtis-Long, M. J.; Seo, W. D.; Jeong, S. H.; Jeong, I.; Park, K. J. Org. Chem. 2008, 73, 2898. http://dx.doi.org/ 10.1021/jo702480y

11. Yang, X. M. ; Zou, X. M.; Fu, Y. Q.; Mou, K.; Fu, G.; Ma, C.; Xu, P. Synth. Commun. 2007, 37,9 . http://dx.doi.org/ 10.1080/00397910600977509

12. Soucek, M.; Urban, J.; Saman, D. Collect. Czech. Chem. Commun. 1990, 55, 761. http://dx.doi.org/ 10.1135/cccc19900761

13. Dullin, A.; Dufrasne, F.; Gelbcke, M.; Gust, R. ChemMedChem 2006, 1, 644. http://dx.doi.org/ 10.1002/cmdc.200600032

14. Fujii, T.; Itaya, T.; Matsubara, S. Chem. Pharm. Bull. 1989, 37, 1758.

15. Yao, H.; Yamamoto, K. Chem. Asian J. 2012, 7, 1542. 
http://dx.doi.org/ 10.1002/asia.201200017

16. Hamada, Y.; Shioiri, T. Chem. Pharm. Bull. 1982, 30, 1921.

17. Otto, A.; Ziemer, B.; Liebscher, J. Eur. J. Org. Chem. 1998, 2667.

http://dx.doi.org/10.1002/(SICI)1099-0690(199811)1998:11<2267::AID-

EJOC2267 $>3.0 . \mathrm{CO} ; 2-\mathrm{E}$

18. Keck, G. E.; Fleming, S.; Nickell, D.; Weider, P. Synth. Commun. 1979, 9, 281. http://dx.doi.org/ 10.1080/00397917908064153

19. Bui, T.; Candeias, N. R.; Barbas III, C. F. J. Am. Chem. Soc. 2010, 132, 5574. http://dx.doi.org/ 10.1021/ja101032j

20. Momiyama, N.; Yamamoto, H. J. Am. Chem. Soc. 2003, 125, 6038. http://dx.doi.org/ 10.1021/ja0296702

21. Peng, J.; Jiang, D.; Lina, W.; Chen, Y. Org. Biomol. Chem. 2007, 5, 1391. http://dx.doi.org/ 10.1039/b701509g

22. Jahn, U.; Hartmann, P.; Dix, I.; Jones, P. J. Eur. J. Org. Chem. 2002, 718. http://dx.doi.org/ 10.1002/1099-0690(200202)2002:4<718::AID-EJOC718>3.0.CO;2-6

23. Kumarn, S.; Shaw, D. M.; Longbottom, D. A.; Lev, S. V. Org. Lett., 2005, 7, 4189. http://dx.doi.org/ 10.1021/o1051577u

24. Davies, S. G.; Smyth, G. D. J. Chem. Soc., Perkin Trans. 1, 1996, 2467. http://dx.doi.org/ 10.1039/P19960002467

25. Li, C.-J. Chem. Rev. 2005, 105, 3095. http://dx.doi.org/ 10.1021/cr030009u

26. Petrier, C.; Luche, J.-L. J. Org. Chem. 1985, 50, 910. http://dx.doi.org/ 10.1021/jo00206a047

27. Wipf, P.; Furegati, M. Org. Lett. 2006, 8, 1901. http://dx.doi.org/ 10.1021/o1060455e

28. T. Shono, Ishifune, M.; Kashimura, S. Chem. Lett. 1990, 449. http://dx.doi.org/ 10.1246/cl.1990.449

29. Mistryukov, E. A.; Peresypkin, A. V.; Mgulin, V. A. Mendeleev Commun., 1993, 3, 242. http://dx.doi.org/ 10.1070/MC1993v003n06ABEH000311

30. Hodgson, D. M.; Talbot, E. P. A.; Clark, B. P. Org. Lett. 2011, 13, 2594. http://dx.doi.org/ 10.1021/ol200711f

31. Santangelo, E. M.; Ilme Liblikas, D. R.; Norin, T.; Unelius, C. R. J. Org. Chem. 2001, 66, 5384. http://dx.doi.org/ 10.1021/jo015592y

32. Sofiyev, V.; Navarro, G.; Trauner, D. Org. Lett. 2008, 10, 149. http://dx.doi.org/ 10.1021/ol702806v

33. Thamapipol, S.; Kundig, E. P. Org. Biomol. Chem., 2011, 9, 7564. http://dx.doi.org/ 10.1039/c1ob06121f 
34. Fischer, D.; Nguyen, T. X.; Trzoss, L.; Dakanali, M.; Theodorakis, E. A. Tetrahedron Lett. 2011, 52, 4920.

http://dx.doi.org/ 10.1016/j.tetlet.2011.07.054

35. Cong, P. Z. Acta Pharmaceutica Sinica, 1983, 18, 215. 